\title{
Risk Factors for Progression to Severe Sepsis or Septic Shock in Patients with Urolith Induced Obstructive Urosepsis
}

\section{Jiadong Cao}

The Second Affiliated Hospital of Guangzhou University of Chinese Medicine

\section{Zhichao Wang}

The Second Affiliated Hospital of Guangzhou University of Chinese Medicine

\section{Youlian Wang}

The Second Affiliated Hospital of Guangzhou University of Chinese Medicine

Hucai Li

The Second Affiliated Hospital of Guangzhou University of Chinese Medicine

\section{Chiming Gu}

The Second Affiliated Hospital of Guangzhou University of Chinese Medicine

\section{Zunguang Bai}

The Second Affiliated Hospital of Guangzhou University of Chinese Medicine

\section{Zhiqiang Chen}

The Second Affiliated Hospital of Guangzhou University of Chinese Medicine

\section{Shusheng Wang}

The Second Affiliated Hospital of Guangzhou University of Chinese Medicine

Songtao Xiang ( $\nabla$ tonyxst@163.com )

The Second Affiliated Hospital of Guangzhou University of Chinese Medicine

\section{Research Article}

Keywords: urolith, urosepsis, severe sepsis, septic shock, risk factors

Posted Date: December 15th, 2020

DOl: https://doi.org/10.21203/rs.3.rs-109284/v1

License: (c) (i) This work is licensed under a Creative Commons Attribution 4.0 International License. Read Full License 


\section{Risk Factors for Progression to Severe Sepsis or Septic Shock in Patients with Urolith Induced Obstructive Urosepsis}

Cao JD ${ }^{\# 1}$, Wang $\mathrm{ZC}^{\# 1}$, Wang $\mathrm{YL}^{2}$, Li HC${ }^{3}$, Gu CM${ }^{1}$, Bai ZG ${ }^{1}$, Chen ZQ ${ }^{1}$, Wang $\mathrm{SS}^{* 1}$ and Xiang $\mathrm{ST}^{* 1}$

1. From Department of Urology, The Second Affiliated Hospital of Guangzhou University of Chinese Medicine, Guangzhou,China;2.From Department of Andrology, The Second Affiliated Hospital of Guangzhou University of Chinese Medicine,Zhuhai,China;3.From Department of Nephrology, The Second Affiliated Hospital of Guangzhou University of Chinese Medicine, Guangzhou,China

\#These authors contributed equally to this work.

* Both authors are correspondingauthors.

Correspondence:Dr. Xiang ST. Department of Urology, The Second Affiliated Hospital of Guangzhou University of Chinese Medicine, Fangcun Branch, 36 Chong'an Street, North Dongjiao Road, Guangzhou 510370, China(telephone: +86 (020) 8149 9399; FAX: +86 (020) 8149 9866; e-mail: tonyxst@163.com);Dr. Wang SS. Department of Urology, The Second Affiliated Hospital of Guangzhou University of Chinese Medicine, 261 Da Tong Road, Guangzhou, 510120, China (telephone: +86 (020) 87351238; FAX: +86 (020) 87351238; e-mail:13503059270@163.com).

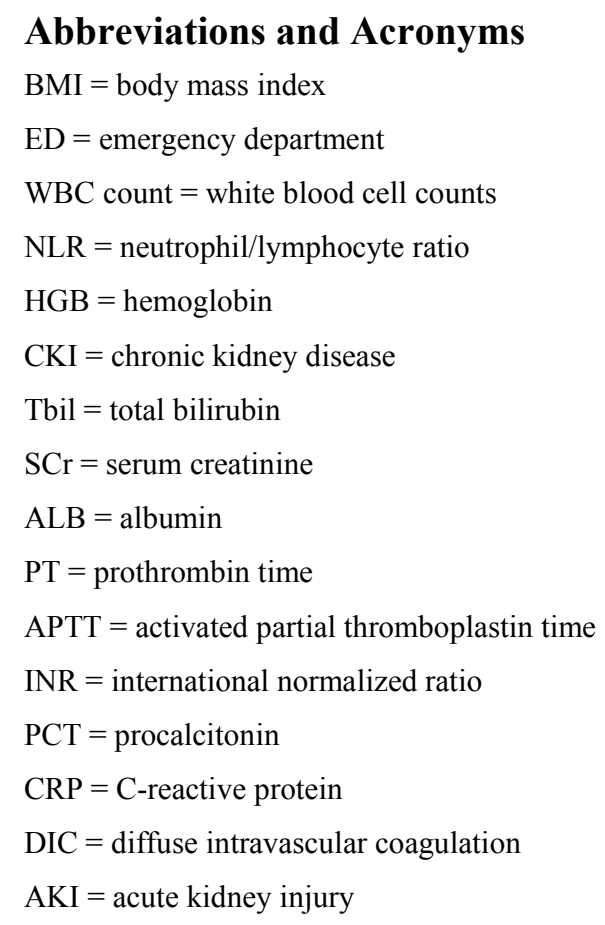

\section{ABSTRACT}

Introduction: To analyze the risk factors for the progression to severe sepsis,or septic shock, in pre-hospital patients with urolith Induced obstructive urosepsis, so as to facilitate the early identification of high-risk patients.

Materials and Methods: Datas were retrospectively reviewed from 160 patients of urolith induced obstructive urosepsis between December 2013 and December 2019. There were 49 patients complicated by severe sepsis (severe sepsis group), 12 patients with septic shock (septic shock group), and 99 patients without progression to severe sepsis or septic shock (sepsis group). The data covered age, gender, BMI, time interval from ED to admission, WBC count, NLR, HGB, etc. Datas were analyzed by univariate analyses and multivariate logistic regression analysis.

Results:Univariate analysis showed that the differences of age, the time interval from ED to admission, history of diabetes mellitus, history of CKI, NLR, HGB, platelet count, TBil, SCr, 
ALB, PT, APTT, INR, PCT, and positive rate of pathogens in blood culture were statistically significant $(\mathrm{P}<0.05)$. Multivariatelogistic regression analysis showed that age, $\mathrm{SCr}$, and history of CKI were independent risk factors for progression to severe sepsis, or septic shock $(\mathrm{P}<0.05)$. Conclusions: Aged $\geq 65$ years, $\mathrm{SCr} \geq 248 \mathrm{~mol} / \mathrm{L}$, and history of $\mathrm{CKI}$ were independent risk factors for progression to severe sepsis, or septic shock, in patients with urolith induced obstructive urosepsis. The key to prevent urosepsis from progression is to improve our understanding of disease dynamics, control the risk factors, and intervene the pre-hospital patients with multidisciplinary modalities as early as possible.

Key words: urolith; urosepsis; severe sepsis; septic shock; risk factors

Urosepsis refers to the systemic inflammatory response caused by urinary tract infection, the onset and progression of which is rapid. People pay more and more attention to urosepsis because the fatality rate is as high as $28.3 \%-41.1 \%$ once it develops into severe sepsis and septic shock. ${ }^{1,2}$ Hoffmann et al. ${ }^{3}$ found that $78 \%$ of patients with urosepsis were caused by obstructive urolith. The research on urosepsis mainly focuses on the risk factors of urosepsis after minimally invasive endoscopic lithotripsy, ${ }^{4,5}$ whereas reports on the pre-hospital risk factors for disease progression from initial diagnosis to more severe conditions are sparse. We analyzed the clinical data in a retrospective multicent cohort study and aimed to evaluate the risk factors for the progression to severe sepsis, or septic shock, in pre-hospital patients with urolith induced obstructive urosepsis, so as to provide insight on early identification of high-risk patients and better clinical outcomes.

\section{MATERIALS AND METHODS}

We retrospectivelyanalyzed the data collected from 160 patients treated with urolith induced obstructive urosepsis between December 2013 andDecember 2019 in three branches of Guangdong Provincial Hospital of Traditional Chinese Medicine. The diagnostic criteria for urosepsis $^{6}$ are as follows: (1) the clinical symptoms caused by urinary tract infection; (2) the systemic inflammatory response syndrome (must fulfill at least two of the following criteria: (1) fever $>38^{\circ} \mathrm{C}$ or hypothermia $<36^{\circ} \mathrm{C}$; (2) tachycardia $>90$ beats/minute; (3) tachypnea $>20$ breaths/minute or the $\mathrm{PCO}_{2}$ of arterial blood was $<32 \mathrm{mmHg}(4.3 \mathrm{kPa})$; (4) leukocytosis $>$ $12 \times 10^{9} / \mathrm{L}$ or leukopenia $<4 \times 10^{9} / \mathrm{L}$, or the ratio of immature white blood cells are $\geq 10 \%$ ). 160 patientswere divided into sepsis group (99 cases), severe sepsis group (49 cases), and septic shock group (12 cases). For the definition of severe sepsis and septic shock, refer to the Surviving Sepsis Campaign: International Guidelines for Management of Sepsis and Septic Shock:2016(2016 SCC Guidelines).

We analyzed the following indicators of the patients: age, gender, BMI, history of diabetes, history of CKI, history of anemia, history of hypertension, history of heart disease, history of cancer, history of urolithiasis surgery, the time interval from ED to admission, the highest temperature level, surface area of stone, stone locations, degree of hydronephrosis, WBC count, NLR, platelet count, HBG, ALB, SCr, TBil, PT, APTT, INR, CRP, PCT, positive rate of pathogens in urine culture, positive rate of urinary nitrite, urinary leukocyte count, and positive rate of pathogens in blood culture.

The data were analyzed by SPSS 21.0 statistical software. Significant variables on univariate analyses were included in a multivariate logistic regression analysis, with statistical significance defined as $\mathrm{P}<0.05$.

All methods were carried out in accordance with relevant guidelines and regulations. And all experimental protocols were approved by Ethics Committee of Guangdong Hospital of Chinese Medicine.All patients were informed and consented to this study, and that subjects are under 18, consent was obtainedfrom a parent and/or legal guardian. 


\section{RESULTS}

A total of 160 patients met the inclusion criteria. The average BMI was $24.29 \mathrm{~kg} / \mathrm{m}^{2}$ (range $16.4-37.6 \mathrm{~kg} / \mathrm{m}^{2}$ ). Gender distribution was 41 male and 119 female, aged 20-92 years (median 63 years). General underlyingdiseases included 34 diabetes, 41 CKIs, 30 anemias, 69 hypertensions, 11 heart diseases, 7 cancers and 46 urolithiasis surgeries. The average time interval from ED to admission was 9.66 hours (range 1-48 hours).

There were significant differences among the sepsis group, the severe sepsis group and the septic shock group in terms of NLR $(\mathrm{P}=0.005)$, platelet count $(\mathrm{P}=0.000), \mathrm{HBG}(\mathrm{P}=0.101), \mathrm{ALB}$ $(\mathrm{P}=0.000), \operatorname{SCr}(\mathrm{P}=0.000)$, Tbil $(\mathrm{P}=0.004), \mathrm{PT}(\mathrm{P}=0.000)$, APTT $(\mathrm{P}=0.000), \mathrm{INR}(\mathrm{P}=0.000)$, PCT $(\mathrm{P}=0.000)$, and positive rate of pathogens in blood culture $(\mathrm{P}=0.001)$. The complete set of results includingthe highest temperature level, WBC count, $\mathrm{CRP}$ etc, is listed below (Table 1). Multivariate logistic regression analysisidentified age $(\mathrm{P}=0.024)$, Scr $(\mathrm{P}=0.000)$, and history of $\mathrm{CKI}(\mathrm{P}=0.002)$ were independent risk factors for progression to severe sepsis, or septic shock, in patients with urolith induced obstructive urosepsis ( Table 2).

Table 1. Clinical characteristics of 160 patients with urolith induced obstructive urosepsis

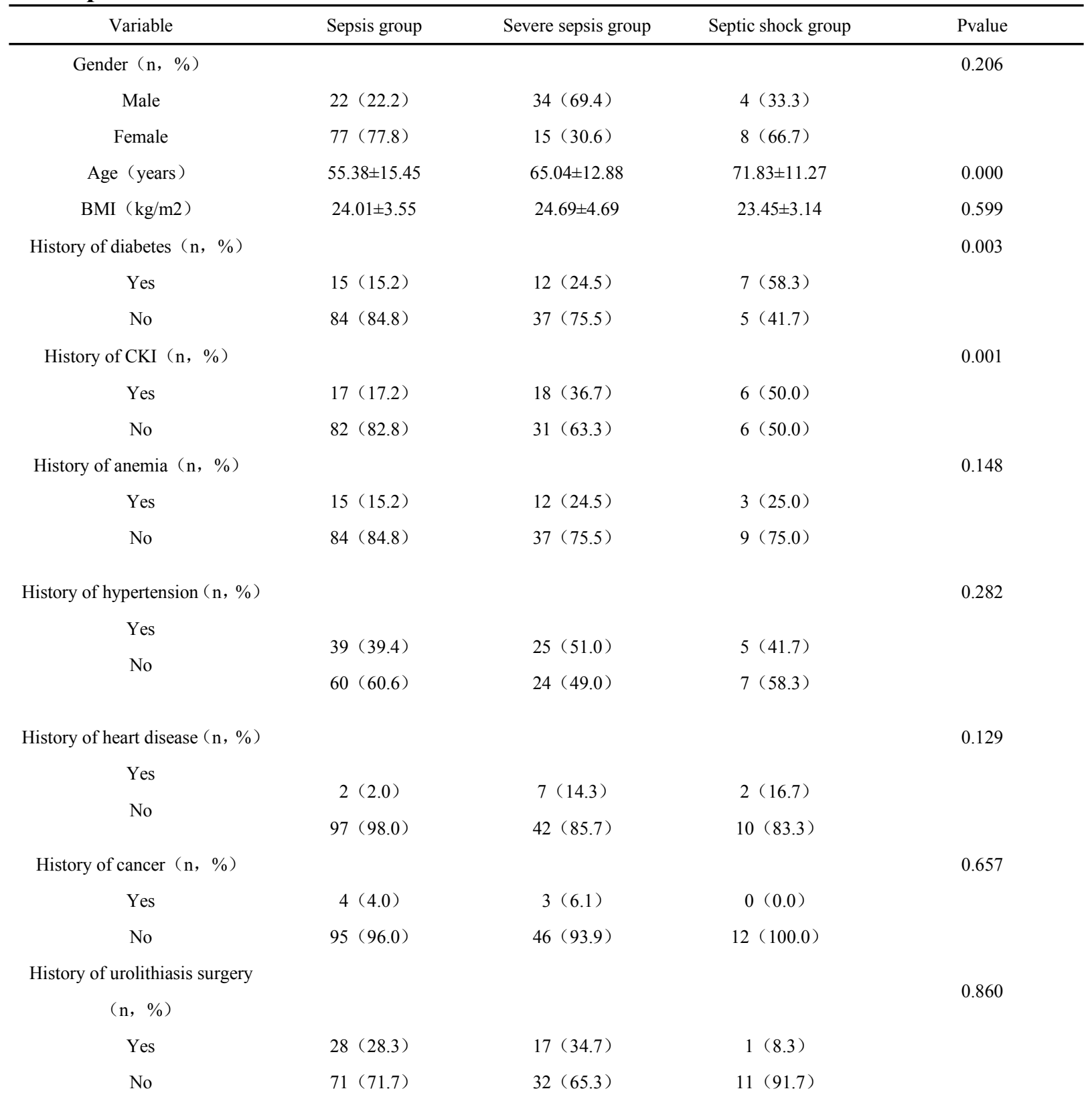




\begin{tabular}{|c|c|c|c|c|}
\hline $\begin{array}{l}\text { Time interval from ED to } \\
\text { admission }(\mathrm{h})\end{array}$ & $8.78 \pm 11.92$ & $10.29 \pm 11.26$ & $13.92 \pm 12.63$ & 0.038 \\
\hline Highest temperature level $\left({ }^{\circ} \mathrm{C}\right)$ & $39.05 \pm 0.57$ & $39.18 \pm 0.74$ & $39.29 \pm 0.62$ & 0.130 \\
\hline Surface area of stones $(\mathrm{cm} 2)$ & $0.79 \pm 1.47$ & $0.51 \pm 0.41$ & $0.45 \pm 0.45$ & 0.102 \\
\hline Laterality of stones $(\mathrm{n}, \%)$ & & & & 0.213 \\
\hline Left & $37(37.4)$ & $28(57.1)$ & $3(25.0)$ & \\
\hline Right & $62(62.6)$ & $21(42.9)$ & $9(75.0)$ & \\
\hline Location of stones $(n, \%)$ & & & & 0.537 \\
\hline Upper & $54(54.5)$ & $30(61.2)$ & $4(33.3)$ & \\
\hline Middle & $14(14.1)$ & $3(6.1)$ & $2(16.7)$ & \\
\hline Lower & $31(31.3)$ & $16(32.7)$ & $6(50.0)$ & \\
\hline $\begin{array}{c}\text { Degree of hydronephrosis } \\
(\mathrm{n}, \%)\end{array}$ & & & & 0.823 \\
\hline Mild & $63(63.6)$ & $27(55.1)$ & $9(75.0)$ & \\
\hline Moderate & $24(24.2)$ & $15(30.6)$ & $3(25.0)$ & \\
\hline Severe & $12(12.1)$ & $7(14.3)$ & $0(0)$ & \\
\hline WBC count $(/ \mathrm{L})$ & $18.09 \pm 5.93$ & $17.97 \pm 8.58$ & $19.11 \pm 8.45$ & 0.857 \\
\hline NLR $(\%)$ & $18.39 \pm 12.63$ & $25.74 \pm 22.20$ & $31.52 \pm 30.32$ & 0.005 \\
\hline Platelet count $(109 / \mathrm{L})$ & $228.18 \pm 73.98$ & $150.73 \pm 89.07$ & $113.50 \pm 99.97$ & 0.000 \\
\hline HBG $(g / L)$ & $121.33 \pm 16.92$ & $114.04 \pm 18.96$ & $104.42 \pm 16.27$ & 0.101 \\
\hline $\operatorname{ALB}(\mathrm{g} / \mathrm{L})$ & $138.94 \pm 4.94$ & $34.18 \pm 5.26$ & $32.60 \pm 5.99$ & 0.000 \\
\hline $\mathrm{SCr}(\mu \mathrm{mol} / \mathrm{L})$ & $103.15 \pm 29.80$ & $248.08 \pm 130.71$ & $498.08 \pm 660.27$ & 0.000 \\
\hline TBil $(\mu \mathrm{mol} / \mathrm{L})$ & $13.58 \pm 6.59$ & $18.38 \pm 18.10$ & $29.61 \pm 39.69$ & 0.004 \\
\hline $\mathrm{PT}(\mathrm{s})$ & $12.77 \pm 1.19$ & $13.47 \pm 1.48$ & $15.32 \pm 3.33$ & 0.000 \\
\hline APTT $(s)$ & $28.83 \pm 4.51$ & $30.42 \pm 5.32$ & $39.96 \pm 14.75$ & 0.000 \\
\hline INR $(R)$ & $1.09 \pm 0.11$ & $1.17 \pm 1.38$ & $1.33 \pm 0.29$ & 0.000 \\
\hline $\mathrm{CRP}(\mathrm{mg} / \mathrm{L})$ & $120.64 \pm 76.38$ & $156.25 \pm 115.68$ & $216.89 \pm 108.08$ & 0.148 \\
\hline PCT $(\mathrm{ng} / \mathrm{mL})$ & $13.51 \pm 22.50$ & $40.40 \pm 38.57$ & $62.94 \pm 45.28$ & 0.000 \\
\hline $\begin{array}{l}\text { Positive rate of pathogens in } \\
\text { urine culture }(n, \%)\end{array}$ & $38(40.9)$ & $19(38.8)$ & $6(50.0)$ & 0.373 \\
\hline $\begin{array}{l}\text { Positive rate of urinary nitrite } \\
\qquad(\mathrm{n}, \%)\end{array}$ & $18(29.5)$ & $16(32.7)$ & $16(32.7)$ & 0.665 \\
\hline Urinary leukocyte count (/ul) & $2499.50 \pm 8818.60$ & $1600.55 \pm 6501.60$ & $3789.90 \pm 7121.70$ & 0.828 \\
\hline $\begin{array}{l}\text { Positive rate of pathogens in } \\
\text { blood culture }(n, \%)\end{array}$ & $11(22.4)$ & $9(36.0)$ & $7(63.6)$ & 0.001 \\
\hline
\end{tabular}

\section{Table 2. Multivariate logistic regression analysis results}

\begin{tabular}{ccccc}
\hline Variable & B & OR & $95 \%$ CI & P Value \\
\hline Age & 0.045 & 1.046 & $0.006 \sim 0.085$ & 0.024 \\
Scr & 0.015 & 1.015 & $0.009 \sim 0.020$ & 0.000 \\
History of CKI & 1.840 & 6.296 & $0.694 \sim 2.985$ & 0.002 \\
\hline
\end{tabular}

Table 3.Constituent ratios of the pathogens (\%)

\begin{tabular}{cccccc}
\hline Pathogens in urine culture & Strains & Constituent ratios $(\%)$ & Pathogens in blood culture & Strains & Constituent ratios $(\%)$ \\
\hline Gram-negative bacteria & 53 & 84.13 & Gram-negative bacteria & 26 & 96.29 \\
Escherichia coli & 41 & 65.08 & Escherichia coli & 22 & 81.48 \\
Klebsiella pneumoniae & 2 & 3.17 & Klebsiella pneumoniae & 3 & 11.10 \\
Proteus mirabilis & 2 & 3.17 & Proteus mirabilis & 1 & 3.71 \\
\hline
\end{tabular}




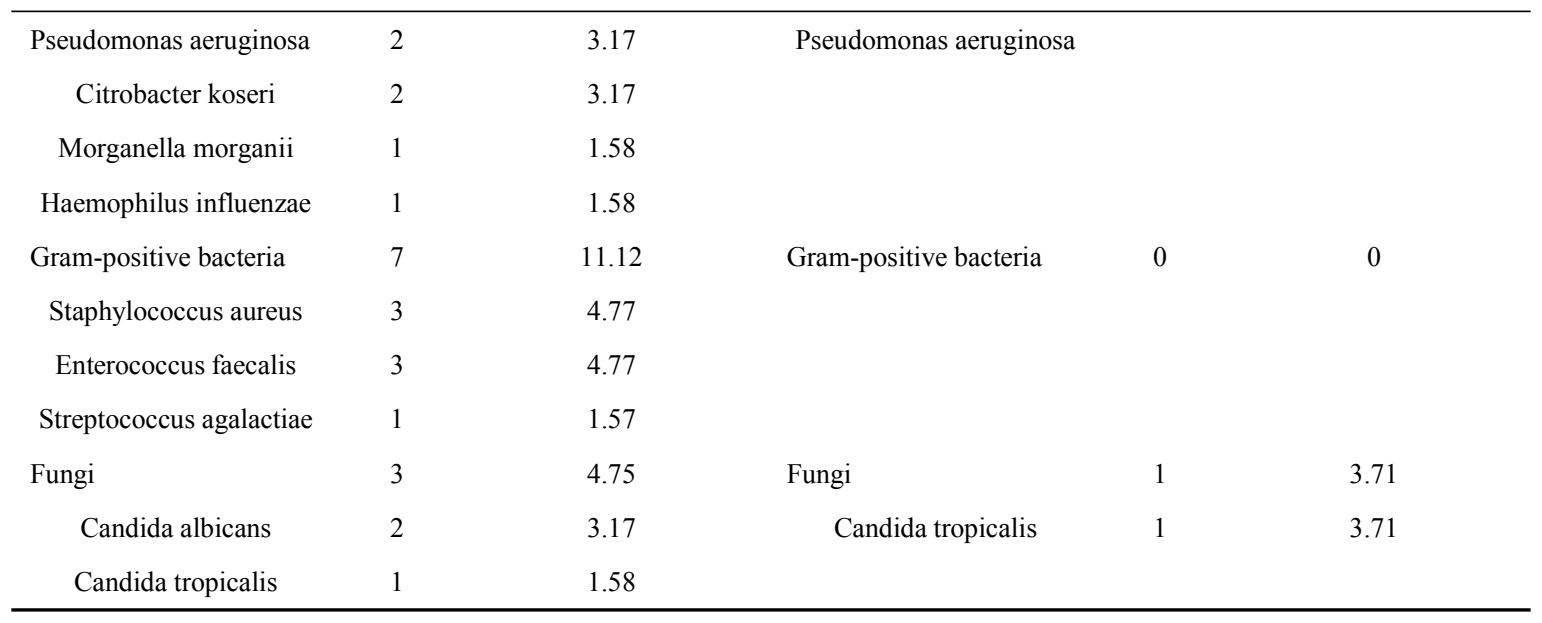

Table 4. Age, Scr, and history of CKI in predicting the progression in patients with urolith induced obstructive urosepsis by ROC curve

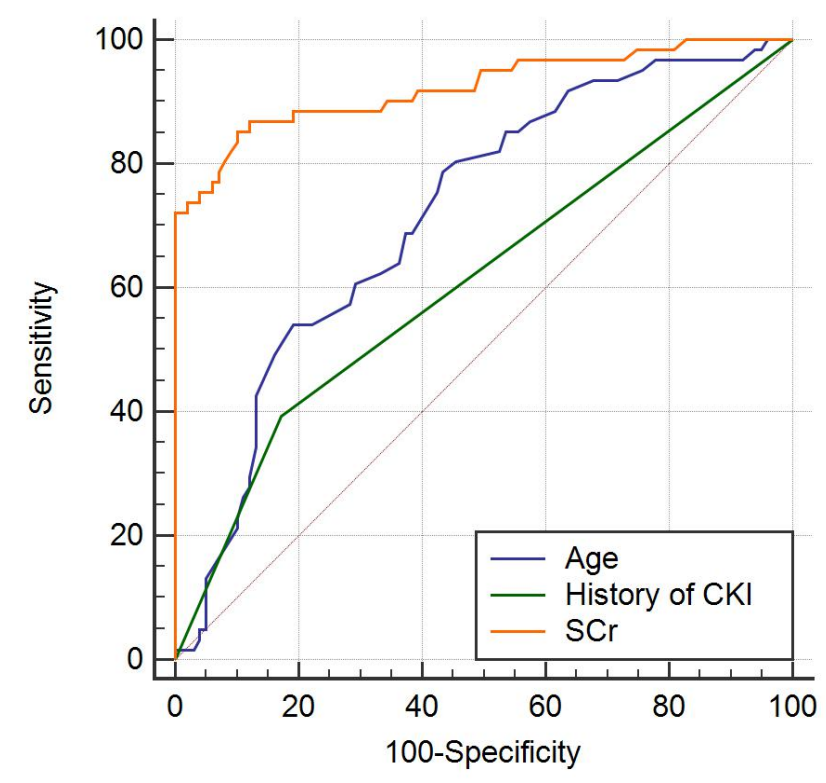

\section{DISCUSSION}

Urolith induced obstructive urosepsis is one of the most common emergencies encountered in urology, which is characterized by its severity and rapid progression. By evaluating risk factors for its progression to severe sepsis, or septic shock, it is particularly important to define significant indicators for clinicians to identify high-risk patients. Of the 160 pre-hospital patients with urolith induced obstructive urosepsis in this study, 61 patients developed severe sepsis, or septic shock, with an incidence of $38.13 \%$. This result supports the notion that obstructive urosepsis has a high risk of progression to more severe conditions and again underscore the necessity and importance of further investigation of its risk factors.

Urolith induced obstructive urosepsis occurs predominantly in female patients, ${ }^{7}$ which also holds true in our study in that the male to female ratio was 1:3.5. The reason is considered to be related to the anatomical characteristics of female lower urinary tract, the higher incidence of female lower urinary tract infection, and the refractory infection of drug-resistant bacteria. However, univariate analysis showed that gender was not a significant risk factor for the progression of urosepsis. The age data of the three groups showed that the average age of the septic shock group was about $71.83 \pm 11.27$ years old, which was much higher than that of the sepsis group and the severe sepsis group ( $55.38 \pm 15.45$ years and $65.04 \pm 12.88$ years). Martin et al. ${ }^{8}$ found that there was a higher mortality rate if septic patients were older and that age was an 
independent risk factor for mortality of septic patients. Studies have indicated that age is a risk factor for the progression to severe sepsis or septic shock. ${ }^{9}$ In patients with CKI, the higher the baseline Scr, the more serious the kidney damages have, the greater imbalances of homeostasis are in patients with sepsis. In addition, it is difficult for antibiotics to reach the collecting system of the affected kidney, which leads to the poor infection control and worse prognosis.

Early and multidisciplinary comprehensive intervention of sepsis are essential to improve the prognosis and reduce the mortality. ${ }^{10,11}$ The 2016 SCC Guidelines emphasizes the concept of "time to antibiotics" and "hour-1 bundle". In this study, we defined the "time" as the interval between the ED reception and hospital admission for specialized treatment. By comparing the data between the groups, significant differences were found $(\mathrm{P}=0.038)$. The earlier the time of specialized intervention, the lower the probability of disease progression is.

The pathogenesis of sepsis is still unclear, which may be closely related to the secretion of inflammatory mediators, immune dysfunction, endotoxin translocation, and other factors. When sepsis occurs, neutrophils are released from the bone marrow into the peripheral circulation and apoptosis is delayed. ${ }^{12}$ Tulzo et al. ${ }^{13}$ showed that the apoptosis of peripheral lymphocyte increased by 5 times in patients with septic shock compared to those with sepsis. Overactivated eutrophils and the decrease of lymphocytes will lead to further suppression of immune function. The NLR reflects the balance between the inflammatory level and the immune status of septic patients, which was significant difference among groups in this study $(\mathrm{P}=0.005)$. Of note, it should be pointed out that there was no significant difference in the level of WBC count $(\mathrm{P}=0.857)$. The WBC count were usually significantly increased in severe infecti on, but significant decrease can also occur. In this study cohort, there were five patients with leukopenia, and the differences were still insignificant after the elimination of the five cases $(\mathrm{P}=0.284)$. Leukopenia is one of the important indicators in the diagnosis of sepsis. The five leukopenic patients in our study accounted for $1.01 \%$ in sepsis group, and $6.55 \%$ in severe sepsis and septic shock group, and the difference was statistically significant $(\mathrm{P}=0.001)$ by the binary logistic regression analysis. It can be concluded thatleukopeniamay be one of the risk factors for the progression to severe sepsis, or septic shock, and should elicit particular clinical attention.

CRP and PCT are widely used in clinical practice as important indicators to reflect the degree of inflammation in the body. Fukashi Yamamichi et al. ${ }^{14}$ analysed on the data of 77 patients with urosepsis, suggesting that CRP was the only risk factor for sepsis involving tumor obstructionto progress to septic shock. In contrast to Yamamichi, most scholars believe that PCT is significantly superior to CRP in the diagnostic sensitivity and specificity of severe sepsis and septic shock, and is also superior to CRP in the assessment of the severity and prognosis of the disease. PCT level is directly proportional to the severity of sepsis caused by bacterial infection, which has important diagnostic significance for bacterial infection. In this study, the average levels of CRP and PCT in patients with severe sepsis, or septic shock, were higher than those in the sepsis group, and the differences of PCT was statistically significant in univariate analysis $(\mathrm{P}=0.000)$. However, the results of multivariatelogistic regression analysis showed no statistical significance.The 2016 SSC guidelines also recommend PCT as an adjunctive indicator in sepsis treatment. However, PCT tests have issues with some false positive and false negative results, ${ }^{15}$ and different pathogens may lead to different up-regulated PCT responses. ${ }^{16}$ As concerned by the SCC guidelines, the PCT can only serve as a supplement in clinical assessment due to the complexity of sepsis and septic shock, therefore the recommendation remains cautious.

Sepsis and septic shock are the main causes of AKI, and more than $50 \%$ of ICU patients with AKI are associated with sepsis. ${ }^{17}$ The incidence of AKI in clinically septic patients is as high as $23 \% .{ }^{18}$ Currently, it is believed that AKI is caused by the toxic effect of soluble inflammatory 
factors released during inflammatory response. ${ }^{19}$ The higher the Scr level, the worse prognosis of patients with urosepsis will have, and it can be used as an independent risk indicator to predict the disease progression and prognosis. ${ }^{20}$ Hypoproteinemia is also common complications of sepsis and related to the severity and prognosis of the disease. Hypoproteinemia can be used as an important indicator to evaluate the prognosis of septic patients: the lower the serum albumin, the lower immunity level and the more severe of the condition are. ${ }^{21}$ The Japanese study confirmed that the decrease of serum albumin can be used to predict the risk of septic shock in patients with acute obstructive pyelonephritis. ${ }^{22}$

The massive release of inflammatory mediators in septic patients activates the coagulation system, which leads to coagulation disturbance and thrombocytopenia. ${ }^{23,24} \mathrm{Kamei}^{25}$ found that thrombocytopenia was high risk factors for severe infection. Severe sepsis often leads to decreased platelet levels and relevant studies have confirmed that thrombocytopenia can be used as a risk factor for predicting the progression of sepsis. ${ }^{26-27}$ Severe infection, DIC, cytophagocytosis, and even immunosuppression in patients with sepsis can lead to excessive consumption of platelets, leading to thrombocytopenia, which may independently alter the patient's immune response to infection. ${ }^{28-29}$ If the platelet level can recover rapidly after treatment, it often indicates a good prognosis, which is supported by a study of prognostic factors for septic patients. ${ }^{30}$

Pathogen culture and drug sensitivity identification are important clinically for the treatment and prognosis. In this study, the collection rates of urine culture (143/160) and blood culture (85/160) were both low, which raises the concern of standard of care and should be improved in the future. In this study, 63 strains of pathogens were isolated in urine culture and a total of 27 strains of pathogens were isolated in blood culture. ( Table 3). The positive rate of blood culture, as the "gold standard", is not high, which may be related to early treatment of broad-spectrum antibiotic. It is worth noting that ideally the blood culture specimens should always be collected prior to the administration of antimicrobial therapy. Current studies have shown that compared to gram-positive bacterial infection, patients with gram-negative bacterial infection are more severely afflicted and are more prone to severe sepsis or septic shock. In this study, gram-negative bacteria were the main culprit in the blood stream of patients with urosepsis and the difference in blood culture positive rate among groups was significant (sepsis group : severe sepsis group : septic shock group $=22.44 \%: 36.00 \%: 63.63 \%$ ). Univariate analysis showed that positive blood culture was a risk factor for sepsis progression, although its contribution was no longer significant in multivariate logistic analysis.

Multivariate logistic regression analysis was performed on the risk factors selected from the above univariate analysis for sepsis progression. The results showed that the differences in age, Scr and history of CKI were statistically significant ( $\mathrm{P}=0.024, \mathrm{P}=0.000, \mathrm{P}=0.002$, respectively). Older age is one of the independent risk factors for the progression of sepsis. According to our study, if the patients are older than 65 years, their systemic inflammatory responses are more intense. Scr level has been used to evaluate the degree of AKI in patients with urosepsis.Patients with urosepsis induced by urolith obstructive may have rapid deterioration of renal function in a short period of time, especially when $\mathrm{SCr} \geq 248 \mathrm{~mol} / \mathrm{L}$, which leads to increased serum concentrations of systemic inflammatory mediators and further aggravates the risk of infection. This phenomenon is more obvious in patients with CKI.For septic patients with chronic renal insufficiency, early intervention to protect renal function is helpful to improve the prognosis and prevent disease progr ession.If the patients who progressed to severe sepsis group and septic shock group were grouped into one group, that is the progressive group (61 cases in total); this study, the datas were simply divided into the sepsis group (99 cases) and the progressive group. 
By comparing the differences in age, Scr, and history of CKI between the two groups, the ROC curve was used to compare the value of these three risk factors in predicting the progression in patients with urolith induced obstructive urosepsis, respectively. The results showed that age, Scr and history of CKI all had good predictive functions. The area under ROC curve was 0.718 , 0.923 and 0.611 , respectively, and the prediction accuracy of Scr was higher than the other two datas.( Table 4).

\section{CONCLUSIONS}

To summarize our findings from this multicenter retrospective cohort study, patients aged $\geq 65$ years, $\mathrm{Scr} \geq 248 \mathrm{~mol} / \mathrm{L}$, and history of CKI were independent risk factors for progression to severe sepsis, or septic shock, inpre-hospital patients with urolith induced obstructive urosepsis. It is essential to halt the progression of urosepsis by identifying its risk factors and to treat it as early as possible. The main limitation of this study lies in that this is a retrospective cohort study, with deficiencies such as incomplete test panels, ambiguous definition of indicators, and inconsistent treatment regimens, which need to be addressed by a better designed prospective study.

\section{DECLARATIONS}

\section{Ethics approval and consent to participate}

Our study has been approved by the Ethics Committee of Guangdong Hospital of Traditional Chinese Medicine. And all patients were informed and consented to this study, and that subjects are under 18, consent was obtained from a parent and/or legal guardian.

\section{Consent for publication}

Not applicable.

\section{Availability of data and materials}

Not applicable.

\section{Competing interests}

Not applicable.

\section{Funding}

Not applicable.

\section{Authors' contributions}

All authors contributed to the review and read and approved the final manuscript. Cao JD wrote the article.

\section{Acknowledgements}

The kind cooperation of urology and andrology and Nephrology of Guangdong hospital of Chinese medcine, and the participants of the study is sincerely appreciated.

\section{REFERENCES}

1.Wagenlehner FME, Pilatz A, Weidner W et al: Urosepsis:overview of the diagnostic and treatment challenges. Microbiol Spectr 2015; 3:3.

2. Levy MM, Artigas A, Phillips GS et al: Outcomes of thesurviving sepsis campaign in intensive care units in the USA andEurope:a prospective cohort study.Lancet Infect Dis2012;12:919.

3. Hofmann W: Urosepsis and uroseptic shock.Z Urol Nephrol1990 ;83:318.

4. Xiang ST,Wang SS,Gan S et al: Diagnosis and therapy of uroseptic shock by percutaneous nephrolithotomy.Chin J Urol2010;31:522.

5. Schroder U C, Bokeloh F, O'Suliivan M, et al.Guidelines on urological infections[J]. Biomicrofluidics, 2015;13: 361. 
6. Rhodes A, Evans LE, Alhazzani W et al: Surviving Sepsis Campaign: international Guidelines for Management of Sepsis and Septic Shock:2016.Intensive Care Med2017;43:330.

7. Hu M, Zhong X, Cui X et al: Development and validation of a riskprediction nomogram for patients with ureteral calculi associated with urosepsis: A retrospective analysis.PLoS One2018 ;13:10.

8. Martin GS, Mannino DM, Moss M:The effect of age on the development and outcome of adult sepis.Crit Care Med2006;34:17.

9. Fowler AA 3rd, Truwit JD, Hite RD et al: Effect of Vitamin C Infusion on Organ Failure and Biomarkers of Inflammation and Vascular Injury in Patients With Sepsis and Severe Acute Respiratory Failure: The CITRIS-ALI Randomized Clinical Trial.JAMA2019;322:1265.

10. Rhodes A, Phillips G, Beale R et al: The surviving sepsis campaign bundles and outcome:results from the International Multicentre Prevalence Study on Sepsis(the IMPreSS study).Intensive Care Med 2015;41:1621.

11. Kaukonen KM, Bailey M, Suzuki S et al: Mortality related to severe sepsis and septic shock among critically ill patients in Australia and New Zealand,2000-2012.JAMA2014;311:1311.

12. Hotchkiss RS,Nicholson DW: Apoptosis and caspase regulate seath and inflammation in sepis.Nat Rev Immunol2006;6:813.

13. Le Tulzo Y, Pangault C, Gacouin A et al: Early circulating lymphocyte apoptosis in human sepis shock is associated with poor outcome.Shock2002;18:488.

14. Yamamichi F, Shigemura K, Kitagawa K et al: Shock due to urosepsis: A multicentre study.Can Urol Assoc J 2017;11:E106.

15. Schuetz P, Christ-Crain M, Müller B: Procalcitonin and other biomarkers to improve assessment and antibiotic stewardship in infections-hope for hype? .Swiss Med Wkly2009;139:324.

16. Krüger S, Ewig S, Papassotiriou J et al: Inflammatory Parameters predict etiologic patterns but not allow for individual prediction of etiology in patients with CAP:results from the German competence network CAPNETZ.Respir Res2009;10:65. 17. Poukkanen M, Vaara ST, Pettilä V et al:Acute kidney injury in patients with severe sepsis in Finnish Intensive Care Units.Acta Anaesthesiol Scand2013;57:870.

18. Gonullu H, Aslan M, Karadas S et al: Serum prolidase enzyme activity and oxidative stress levels in patients with acute hemorrhagic stroke. Scand J Clin Lab Invest2014;74:200.

19. Dellepiane S, Marengo M, Cantaluppi V: Detrimental cross-talk between sepsis and acute kidney injury: new pathogenic mechanisms, early biomarkers and targeted therapies.Crit Care2016;20:1.

20. Feng ZZ: Analyse Of The Related Factors Which Influening The Prognosis Sepisis. Nan Ning: Guangxi Medical University2016:9.

21. Leite HP, Rodrigues da Silva AV, de Oliveira Iglesias SB et al: Serum Albumin is an independent predictor of clinical outcomes in critically Ill children.Pediatr Crit Care Med2016;17:e56.

22. Tambo M, Okegawa T, Shishido T et al: Predictors of septicshock in obstructive acute pyelonephritis. World J Urol2014;32:803.

23. Fitch SJ, Gossage JR: Optimal management of septic shock - Rapid recognition and institution of therapy are crucial. Postgrad Med2002;111:64.

24. Court O, Kumar A, Parrillo JE et al: Clinical review:myocardial depression in sepsis and septic shock. Crit Care $2002 ; 6: 500$.

25. Kamei J, Nishimatsu H, Nakagawa $\mathrm{T}$ et al: Risk factors for septic shock in acute obstructive pyelonephritis requiring emergency drainage of the upper urinary tract.Int Urol Nephrol2014;46:493.

26. Ogura H, Gando S, Iba T et al: SIRS-associated coagulopathy and organ dysfunction in critically ill patients with thrombocytopenia.Shock2007;28:411.

27. Iba T, Gando S, Murata A et al: Predicting the severity of systemic inflammatory response syndrome (SIRS)-associated coagulopathy with hemostatic molecular markers and vascular endothelial injury markers.J Trauma2007 ;63:1097.

28. Claushuis TA, van Vught LA, Scicluna BP et al: Thrombocytopenia is associated with a dysregulated host response in critically ill sepsis patients.Blood2016;127:3062. 
29. Lee JH, Lee YM, Cho JH: Risk factors of septic shock in bacteremic acute pyelonephritis patients admitted to an ER.J Infect Chemother2012;18:133.

30. Ni T: The analysis of clinical features and riskfactors in sepsis-associatedthrombocytopenia. He Fei:Anhui Medical University2019:19. 


\section{Supplementary Files}

This is a list of supplementary files associated with this preprint. Click to download.

- DATA.xIsx

- supplementaryinformation.docx 